\title{
Fósforo remanescente em solos formados sob diferentes materiais de origem em três topossequências, Pinheiral- $\mathbf{R J}^{1}$
}

\section{Remaining phosphorus in soils developed from different parent materials in three topossequences, Pinheiral-RJ}

\author{
Ademir Fontana ${ }^{2 *}$; Marcos Gervasio Pereira ${ }^{3}$; Adailde Carmo dos Santos ${ }^{4}$; \\ Thiago Andrade Bernini ${ }^{5}$; Lúcia Helena Cunha dos Anjos ${ }^{3}$; \\ Carlos Fernando Dorronsoro Fernández ${ }^{6}$; Francisco José Martins Peinado ${ }^{6}$
}

\section{Resumo}

Este trabalho teve o objetivo de avaliar os teores do fósforo remanescente e correlacioná-los com os atributos químicos, físicos e mineralógicos em horizontes de solos formados a partir de diferentes materiais de origem em três topossequências no município de Pinheiral, RJ. Foram descritos e coletadas amostras de horizontes superficiais (A) e subsuperficiais $(B+C)$ de 14 perfis de solos sob pastagem em três topossequências (T1, T2 e T3), sendo T1 sob basalto, T2 sob muscovita-biotita-gnaisse e T3 sob gabro. Foram realizadas as análises químicas e físicas de rotina, bem como $\mathrm{Al}$ e Fe pelo ataque sulfúrico e fluorescência de raios X, Fed (ditionito-citrato-bicarbonato de sódio) e Feo (oxalato ácido de amônio), superfície específica da argila (SE), mineralogia da argila e óxido, e fósforo remanescente (Prem). Variações dos teores de Prem foram observadas, as quais relacionadas à natureza do material de origem, posição na paisagem e grau de desenvolvimento dos solos, o que pode ser analisado pelas variações dos atributos químicos, físicos e mineralógicos. Os teores de Prem seguiram a ordem T2 > T1 = T3 no horizonte A e da T1 > T3 e T2 T1 e T3 nos horizontes B+C. Foram observadas correlações negativas entre os teores de Prem com $\mathrm{Fe}_{2} \mathrm{O}_{3}\left(\right.$ raios X) $>\mathrm{Fed}>\mathrm{SE}>$ Feo nos horizontes A e com $\mathrm{Fe}_{2} \mathrm{O}_{3}$ (ataque sulfúrico $)>\mathrm{Fe}_{2} \mathrm{O}_{3}($ raios $\mathrm{X})>$ argila $>\mathrm{Fed}>\mathrm{Feo}$ nos horizontes $\mathrm{B}+\mathrm{C}$. As análises de componentes principais e agrupamento hierárquico contribuíram para avaliação conjunta dos dados, evidenciando os atributos relacionados e as similaridades entre os solos.

Palavras-chave: Adsorção de fosfato, horizontes superficiais e subsuperficiais, atributos dos solos

\begin{abstract}
This work aimed to evaluate the remaining phosphorus tenors and correlate with the chemical, physical and mineralogical attributes in soils horizons developed from different parent materials in three topossequences in the municipality of Pinheiral, RJ. Were described and collected samples of surface
\end{abstract}

1 Parte da Tese de Doutorado do terceiro autor apresentada ao Curso de Pós-Graduação em Agronomia, Ciência do Solo da Universidade Federal Rural do Rio de Janeiro. BR 465 Km 7, Seropédica, RJ.

2 Pesquisador A, Embrapa Solos, Rio de Janeiro, RJ. E-mail: ademir.fontana@embrapa.br

3 Profs. Associado IV, Depto de Solos, UFRRJ, Seropédica, RJ. Bolsista da FAPERJ e CNPq. E-mail: gervasio@ufrrj.br; lanjos@ ufrrj.br

4 Prof Instituto Federal de Educação, Ciência e Tecnologia da Bahia, Campus Valença, Valença, BA. E-mail: adacsantos@gmail. com

5 Eng $^{\mathrm{o}}$ Agr $^{\mathrm{o}}$, Instituto Federal de Educação, Ciência e Tecnologia do Rio de Janeiro, Campus Pinheiral, Pinheira, RJ. E-mail: thiagoagronomo@ibest.com.br

6 Profs. do Dept ${ }^{\circ}$ de Edafología y Química Agrícola- Facultad de Ciencias, Universidad de Granada, Fuente Nueva, Granada, Espanha. E-mail: cfdorron@ugr.es; fjmartin@ugr.es

Autor para correspondência 
horizons (A) and subsurface $(\mathrm{B}+\mathrm{C})$ from 14 soils profiles under pasture in three toposequences $(\mathrm{T} 1$, $\mathrm{T} 2$ and $\mathrm{T} 3$ ), being $\mathrm{T} 1$ under basalt, $\mathrm{T} 2$ under muscovite-biotite-gneiss and $\mathrm{T} 3$ under gabbro. Chemical and physical routine analyses as well as $\mathrm{Al}$ and Fe by sulfuric acid attack and X-ray fluorescence, Fed (dithionite-citrate-bicarbonate) and Feo (ammonium oxalate), clay specific surface (SS), clay and oxide mineralogy and remaining phosphorus (Prem), were taken. Variations in the Prem tenors were observed, which related to the nature of parent material, landscape position and soils development degree, which can be analyzed by the chemical, physical and mineralogical variations. The Prem tenors followed the order $\mathrm{T} 2>\mathrm{T} 1=\mathrm{T} 3$ in $\mathrm{A}$ horizons and $\mathrm{T} 1>\mathrm{T} 3$ and $\mathrm{T} 2 \sim \mathrm{T} 1 \mathrm{e} \mathrm{T} 3$ in $\mathrm{B}+\mathrm{C}$ horizons. Negative correlations between Prem tenors with $\mathrm{Fe}_{2} \mathrm{O}_{3}\left(\mathrm{X}\right.$ rays) $>\mathrm{Fed}>\mathrm{SS}>\mathrm{Feo}$ in the A horizon and with $\mathrm{Fe}_{2} \mathrm{O}_{3}$ (sulfuric acid) $>\mathrm{Fe}_{2} \mathrm{O}_{3}(\mathrm{X}$ rays $)>$ clay $>$ Fed $>\mathrm{Feo}$ in the $\mathrm{B}+\mathrm{C}$ horizons, were observed. The principal components and hierarchical clustering analysis contributed to the joint data evaluation, showing the related attributes and the similarity between soils.

Key words: Phosphate adsorption, surface and subsurface horizons, soils attribute

\section{Introdução}

Os solos de regiões tropicais são conhecidos pela elevada capacidade de adsorção específica de ânions fosfato (fixação de fósforo) das fontes solúveis de fertilizantes, deixando-o indisponível e gerando déficit deste nutriente ao longo do ciclo de cultivo de culturas pela redução da concentração de fósforo na solução do solo. Dessa forma são necessárias adubações para sanar as possíveis deficiências nutricionais decorrentes desse fenômeno.

A adsorção de fosfato nestes solos ocorre por meio de troca de ligantes por íons fosfato em solução nas argilas silicatadas e, principalmente, na superfície dos óxidos e hidróxidos, formando complexos de esfera interna, não trocáveis (PARFITT, 1978; SPOSITO, 1989; ALCARDE; GUIDOLIN; LOPES, 1991). Este fenômeno é favorecido pelas características dos minerais da fração argila, como a composição por óxidos e hidróxidos de ferro e alumínio, que apresentam cargas de superfície variáveis segundo a reação da solução do solo, estando carregadas positivamente na maioria dos solos oxídicos em regiões de clima tropical (SPOSITO, 1989).

Assim, a magnitude deste fenômeno é influenciada pela natureza e quantidade dos sítios de adsorção, sendo estes relacionados aos teores de argila, composição mineralógica das frações argila e óxidos, a superfície específica, ao grau de cristalinidade e ao teor de matéria orgânica e frações húmicas (FONTES; WEED, 1996; ANDRADE et al., 2003; ALMEIDA; TORRENT; BARRÓN, 2003; ROLIM NETO et al., 2004; ANTELO et al., 2007; FONTANA et al., 2008; GONÇALVES et al., 2011).

Diante da indisponibilização do fosfato solúvel, estudos tem se desenvolvido com solos brasileiros visando avaliar a capacidade máxima de adsorção e do fósforo remanescente (ALVAREZ et al., 2000; VALLADARES; PEREIRA, ANJOS, 2003; ROLIM NETO et al., 2004; SOUZA et al., 2006; VILAR et al., 2010; GONÇALVES et al., 2011). Essa determinação proposta por Bache e Williams (1971) apresenta-se como uma solução prática para se obter um índice do fator capacidade de adsorção de fosfato dos solos. Nesta proposta, a quantidade de fósforo que permanece na solução é dependente da atuação combinada da concentração de fosfato adicionada e do tempo de contato (ALVAREZ; FONSECA, 1990) e da capacidade de adsorção dos solos (NOVAIS; SMYTH, 1999).

Sob o aspecto da capacidade de adsorção de cada solo, os atributos relacionados podem variar amplamente entre diferentes solos, sendo reflexo principalmente de características tais como: composição química e mineralógica, textura, e natureza do material de origem (sedimentos ou rochas). Neste sentido, Valladares, Pereira e Anjos (2003) observaram que solos originários de rochas de natureza basáltica apresentaram maior capacidade de adsorção de fósforo, estando essa relacionado com atributos como a superfície específica, os teores de argila, $\mathrm{Al}_{2} \mathrm{O}_{3}$, Fed e Feo. Gonçalves et al. (2011) 
observaram que solos formados dos sedimentos do intemperismo do basalto apresentaram maior capacidade de adsorção de fósforo em relação aqueles formados de sedimentos de granito e arenito. Os autores verificaram que as variações na capacidade de adsorção estavam relacionadas aos teores $\mathrm{Fe}_{2} \mathrm{O}_{3}$, Fed, Feo e argila.

A partir do exposto, esse estudo teve como objetivo avaliar os teores do fósforo remanescente e correlacioná-los com os atributos químicos, físicos e mineralógicos em horizontes de solos formados a partir de diferentes materiais de origem em três topossequências no município de Pinheiral, RJ.

\section{Material e Métodos}

A área de estudo situa-se na região do Médio Paraíba do Sul, município de Pinheiral - RJ, entre as latitudes $22^{\circ} 29^{\prime} 03^{\prime \prime}$ e $22^{\circ} 35^{\prime} 27^{\prime}$ ' S, e longitudes $43^{\circ} 54^{\prime} 49^{\prime \prime}$ e $44^{\circ} 04^{\prime} 05^{\prime \prime} \mathrm{W}$, com altitude variando entre 343 a $547 \mathrm{~m}$. Foram coletadas amostras de horizontes superficias (A) e subsuperficias (B e C) de trincheiras abertas no topo, terço superior, terço médio, terço inferior e baixada, de topossequências (T1, T2 e T3) formadas a partir de diferentes tipos de material de origem em áreas de pastagem nativa. Para a T1 tem-se como material de origem, rochas ígnea extrusiva básica (basalto) ou sedimentos oriundos do intemperismo desta. A T2 é formada por rocha metamórfica ácida (muscovita-biotitagnaisse) ou sedimentos desta, e a T3, originada a partir de rocha intrusiva básica (gabro).

Os perfis foram descritos e coletados segundo Santos et al. (2005). As amostras foram coletadas, secas ao ar, destorroadas e passadas por peneira de 2,00 $\mathrm{mm}$ de malha, obtendo-se a terra fina seca ao ar (TFSA) na qual foram realizadas as análises. Foram determinados o teor de carbono orgânico total (COT); $\mathrm{pH}$ em água e $\mathrm{KCl}$; cátions trocáveis $\left(\mathrm{Ca}^{2+}, \mathrm{Mg}^{2+}, \mathrm{K}^{+}+, \mathrm{Na}^{+}, \mathrm{Al}^{3+}\right)$; fósforo assimilável $(\mathrm{P})$; acidez potencial $(\mathrm{H}+\mathrm{Al})$ e calculada a soma de bases (SB), capacidade de troca catiônica a pH 7,0 (CTC) e a saturação por bases (V\%). Também foi determinada a composição granulométrica (teores de areia, silte e argila) e teores de $\mathrm{Al}_{2} \mathrm{O}_{3}$ e $\mathrm{Fe}_{2} \mathrm{O}_{3}$ pelo ataque sulfúrico 1:1 (EMBRAPA, 1997).

Os teores de $\mathrm{Al}_{2} \mathrm{O}_{3}$ e $\mathrm{Fe}_{2} \mathrm{O}_{3}$ por fluorescência de raios $\mathrm{X}$ foram obtidos a partir da TFSA previamente tratadas com 5,4 g de tetraborato de lítio. Seguiuse com aquecimento a $1.100{ }^{\circ} \mathrm{C}$ até fundirem-se e formarem pastilhas, usando prensa hidráulica. As pastilhas foram analisadas em espectrômetro sequencial de longitude de onda dispersiva Philips Magix Pro (PW-2440), com gerador de raios $\mathrm{X}$ de ânodo RH operando a $4 \mathrm{kw}$ (HALLETT; KYLE, 1993) e quatro filtros de alumínio de 200 e $750 \mu$, bronze de $300 \mu$ e chumbo. As curvas de calibração foram obtidas com amostras-padrão. Para calcular as perdas de carbonato, uma amostra de solo foi calcinada a $1.000{ }^{\circ} \mathrm{C}$ em mufla durante 1 hora. Os resultados foram interpretados por programa analítico para análises quantitativas e semiquantitativas IQ+.

Os teores de Fed e Feo foram determinados na TFSA após extração com solução de ditionitocitrato-bicarbonato de sódio, segundo Mehra e Jackson (1960), modificado por Schulze (1984) e com solução de oxalato ácido de amônio, na ausência de luz, de acordo com Schwertmann (1964).

A superfície específica foi quantificada seguindo as medidas de adsorção de moléculas polares (éter monoetílico de etileno glicol - EMEG) e da superfície específica teórica de determinados minerais de argila tomados como padrão (caulinita e montmorilonita) (DYAL; HENDRICKS, 1950; HEILMAN; CARTER; GONZALEZ, 1965). A amostra de solo (1 g) foi previamente seca em estufa de $110^{\circ} \mathrm{C}$ por 24 horas, seguindo para dessecador conectado a uma bomba pneumática, contendo $\mathrm{CaCl}_{2}$ e EMEG. Foi aplicado vácuo por meio de uma bomba, durante 45 minutos, sendo esta operação repetida até a obtenção da constância de peso da amostra (EMBRAPA, 1997).

Para a análise mineralógica da fração argila foi realizada a difração de raios $\mathrm{X}(\mathrm{DRX})$ em lâminas 
orientadas, montadas com suspensão de argila total $(<2,0 \mu \mathrm{m})$ e obtidas por sedimentação após dispersão com $\mathrm{NaOH} 1 \mathrm{~mol} \mathrm{~L}^{-1}$, sem eliminação de óxidos de ferro (EMBRAPA, 1997). A caracterização mineralógica foi realizada em difratômetro Bruker AXS, modelo D8 Advance, empregando-se radiação de $\mathrm{K} \alpha$ de $\lambda$ 0,015405 $\mathrm{nm}$, produzida por tubo de cobre, $40 \mathrm{kv}$ e $40 \mathrm{~mA}$, com amplitude de varredura igual a $1^{\circ} 2 \theta$ por minuto $\left(0,04^{\circ} / \mathrm{s}\right)$. Para as amostras com indicação de presença de minerais $2: 1$, foram feitos os pré-tratamentos de aquecimento a $550^{\circ} \mathrm{C}$, saturação com etilenoglicol e dimetilsulfóxido, com posterior irradiação pelos raios $\mathrm{X}$.

Para obtenção da fração óxidos de ferro, a fração argila foi submetida ao tratamento com $\mathrm{NaOH} 5$ mol L ${ }^{-1}$ para obtenção da fração rica em óxidos de ferro (Fe-concentrada), segundo Norrish e Taylor (1961), modificado por Kämpf e Schwertmann (1982). A caracterização mineralógica foi realizada em amostras em pó não orientado em difratômetro Bruker AXS, modelo D8 Advance, com radiação de $\mathrm{K} \alpha$ de $\lambda$ 0,015405 $\mathrm{nm}$ e tubo de cobre, $40 \mathrm{kv}$ e $40 \mathrm{~mA}$, com amplitude de varredura igual a $1^{\circ} 2 \theta$ por minuto. As interpretações qualitativas e quantitativas dos difratogramas foram obtidas mediante a utilização do programa de identificação mineral XPowder versão 2004.

O fósforo remanescente (Prem) foi determinado utilizando-se um volume de $5 \mathrm{~cm}^{3}$ de TFSA, sendo este colocado em contato por 1 hora com solução de $\mathrm{CaCl}_{2}$ 0,01 mol L-1 contendo $50 \mathrm{mg} \mathrm{L}^{-1}$ de fósforo (ALVAREZ V.; FONSECA, 1990). Após a homogeneização realizou-se filtragem e na solução de equilíbrio foi determinada a concentração de fósforo utilizando-se o método do ácido ascórbico, modificado por Braga e Defelipo (1972). Com base nos teores de fósforo remanescente (Prem), cada perfil foi classificado em relação à capacidade de adsorção de fosfato, sendo: $\mathrm{MB}=$ muito baixa (46$\left.60 \mathrm{mg} \mathrm{L}^{-1}\right) ; \mathrm{B}=$ baixa (31-45 $\left.\mathrm{mg} \mathrm{L}^{-1}\right) ; \mathrm{M}=$ média (21$\left.30 \mathrm{mg} \mathrm{L}^{-1}\right) ; \mathrm{A}=$ alta $\left(11-20 \mathrm{mg} \mathrm{L}^{-1}\right) ; \mathrm{MA}=$ muito alta (6-10 $\left.\mathrm{mg} \mathrm{L}^{-1}\right) ; \mathrm{EA}=$ extremamente alta $\left(0-5 \mathrm{mg} \mathrm{L}^{-1}\right)$ (ALVAREZ V. et al., 2000).
Os resultados foram submetidos à análise de correlação de Pearson entre o Prem e os atributos dos solos, além de análises de variâncias e comparações das médias pelo teste $\mathrm{F}$ e Tukey. Os teores de COT; pH em água e $\mathrm{KCl}$; $\mathrm{Ca}^{2+}, \mathrm{Mg}^{2+}, \mathrm{Al}^{3+}$; CTC; argila; P; Prem; $\mathrm{Al}_{2} \mathrm{O}_{3}$ e $\mathrm{Fe}_{2} \mathrm{O}_{3}$ (raios X); Fed e Feo; SE, foram selecionados para realização das análises multivariadas. Para a análise de componentes principais foi feita a ordenação dos atributos em dois eixos ortogonais de acordo com a variância total dos atributos, e, para a análise de agrupamento hierárquico a distribuição dos perfis/horizontes em dendograma de similaridade. Para a análise de agrupamento hierárquico foi utilizado o método de agrupamento pareado igualmente ponderado e como medida de similaridade a distância euclidiana. Ambas as análises multivariadas foram realizadas pelo programa Statistica 7.0.

\section{Resultados e Discussão}

A partir dos teores de fósforo remanescente (Prem), os horizontes dos perfis de cada topossequência foram classificados nas diferentes classes de capacidade de adsorção de fosfato (ALVAREZ V. et al., 2000). Os horizontes A foram classificados variando de "baixa a alta" capacidade de adsorção e os horizontes $\mathrm{B}+\mathrm{C}$ de "'alta a extremamente alta" (Tabela 1). Destacam-se variações específicas principalmente no horizonte A do perfil T3P1 e com ausência de um padrão definido para os horizontes $\mathrm{B}+\mathrm{C}$ da $\mathrm{T} 1$ e $\mathrm{T} 2$.

As variações dos teores de Prem observadas entre os horizontes $\mathrm{A}$ e $\mathrm{B}+\mathrm{C}$ nas topossequências indicam padrões diferenciados na adsorção específica de fósforo. Estas variações estão relacionadas à natureza do material de origem, posição na paisagem, grau de desenvolvimento dos solos, o que pode ser analisado pelas variações dos atributos químicos, físicos e mineralógicos. Considerações semelhantes foram verificadas por Rolim Neto et al. (2004) em estudo com solos desenvolvidos sob rochas básicas-alcalinas e ultrabásicas no Alto 
Paranaíba, onde os autores observaram distribuição errática dos teores de Prem nos horizontes B de três topossequências, atribuindo como principais fatores para as variações observadas a textura, dos atributos químicos e mineralógicos dos horizontes estudados.

Tabela 1. Classes de solos e classificação dos horizontes A e B+C de acordo com os teores de fósforo remanescente (Prem).

\begin{tabular}{|c|c|c|c|}
\hline \multirow{2}{*}{ Topo/Perfil } & \multirow{2}{*}{ Classificação Taxonômica dos Solos } & $\mathbf{A}$ & $\mathbf{B}+\mathbf{C}$ \\
\hline & & \multicolumn{2}{|c|}{$\mathrm{mg} \mathrm{L}^{-1}-$} \\
\hline T1P1 & Nitossolo Háplico Distrófico típico & $24(\mathrm{M})$ & 5 (EA) \\
\hline T1P2 & Latossolo Vermelho-Amarelo Distrófico típico & $21(\mathrm{M})$ & 10 (MA) \\
\hline T1P3 & Latossolo Vermelho-Amarelo Distrófico típico & $20(\mathrm{~A})$ & $11(\mathrm{~A})$ \\
\hline $\mathrm{T} 1 \mathrm{P} 4$ & Latossolo Vermelho-Amarelo Distrófico típico & $25(\mathrm{M})$ & $12(\mathrm{~A})$ \\
\hline T1P5 & Gleissolo Háplico Tb Distrófico típico & $20(\mathrm{~A})$ & $23(\mathrm{M})$ \\
\hline $\mathrm{T} 2 \mathrm{P} 1$ & Cambissolo Háplico Tb Distrófico típico & $30(\mathrm{M})$ & $23(\mathrm{M})$ \\
\hline $\mathrm{T} 2 \mathrm{P} 2$ & Argissolo Vermelho-Amarelo Distrófico típico & $31(\mathrm{~B})$ & $16(\mathrm{~A})$ \\
\hline $\mathrm{T} 2 \mathrm{P} 3$ & Cambissolo Háplico Tb Distrófico típico & $29(\mathrm{M})$ & $13(\mathrm{~A})$ \\
\hline $\mathrm{T} 2 \mathrm{P} 4$ & Argissolo Amarelo Eutrófico típico & $28(\mathrm{M})$ & 9 (MA) \\
\hline $\mathrm{T} 2 \mathrm{P} 5$ & Gleissolo Háplico Tb Distrófico típico & $29(\mathrm{M})$ & $24(\mathrm{M})$ \\
\hline $\mathrm{T} 3 \mathrm{P} 1$ & Nessolo Regolítico Eutrófico típico & $25(\mathrm{M})$ & - \\
\hline T3P2 & Chernossolo Argilúvico Órtico saprolítico & $18(\mathrm{~A})$ & 4 (EA) \\
\hline T3P3 & Chernossolo Argilúvico Órtico saprolítico & $13(\mathrm{~A})$ & 7 (MA) \\
\hline Т3P4 & Chernossolo Argilúvico Órtico saprolítico & $17(\mathrm{~A})$ & 7 (MA) \\
\hline
\end{tabular}

$\mathrm{MB}=$ muito baixa (46-60 $\left.\mathrm{mg} \mathrm{L}^{-1}\right) ; \mathrm{B}=$ baixa $\left(31-45 \mathrm{mg} \mathrm{L}^{-1}\right) ; \mathrm{M}=$ média $\left(21-30 \mathrm{mg} \mathrm{L}^{-1}\right) ; \mathrm{A}=$ alta $\left(11-20 \mathrm{mg} \mathrm{L}{ }^{-1}\right) ; \mathrm{MA}^{-}$muito alta (6-10 $\left.\mathrm{mg} \mathrm{L}^{-1}\right) ; \mathrm{EA}=$ extremamente alta $\left(0-5 \mathrm{mg} \mathrm{L}^{-1}\right)$.

Fonte: ALVAREZ V. et al. (2000)

* Topo = topossequência.

Fonte: Elaboração dos autores

Tabela 2. Comparação de médias dos teores de fósforo assimilável (P) e remanescente (Prem) para os horizontes superficiais $(A)$ e subsuperficiais $(B+C)$.

\begin{tabular}{|c|c|c|c|c|c|}
\hline \multirow{3}{*}{ Topossequência } & \multirow{3}{*}{ Material de Origem } & \multicolumn{2}{|c|}{$\mathbf{P}$} & \multicolumn{2}{|c|}{ Prem } \\
\hline & & $\mathrm{A}$ & $\mathrm{B}+\mathrm{C}$ & A & $\mathrm{B}+\mathrm{C}$ \\
\hline & & \multicolumn{2}{|c|}{$-\mathrm{mg} \mathrm{kg}^{-1}-$} & \multicolumn{2}{|c|}{$-\mathrm{mg} \mathrm{L}^{-1}-$} \\
\hline Total & & 4 & 3 & $24 \mathrm{a}$ & $13 \mathrm{~b}$ \\
\hline $\mathrm{T} 1$ & Rocha básica (basalto) & 5 & $7 \mathrm{~A}$ & $22 \mathrm{Ba}$ & $12 \mathrm{ABb}$ \\
\hline $\mathrm{T} 2$ & Rocha ácida (muscovita-biotita-gnaisse) & 2 & $1 \mathrm{~B}$ & $29 \mathrm{Aa}$ & $17 \mathrm{Ab}$ \\
\hline $\mathrm{T} 3$ & Rocha básica (gabro) & 5 & $1 \mathrm{~B}$ & $18 \mathrm{Ba}$ & $6 \mathrm{Bb}$ \\
\hline
\end{tabular}

* Letras iguais e maiúsculas na mesma coluna não diferem estatisticamente pelo teste Tukey a $\mathrm{p}<0,01$ nos horizontes A e a $\mathrm{p}<0,08$ nos horizontes $\mathrm{B}+\mathrm{C}$.

* Letras iguais e minúsculas na mesma linha não diferem estatisticamente pelo teste $\mathrm{F}$ a $\mathrm{p}<0,05$.

Fonte: Elaboração dos autores. 
No que se refere aos teores de fósforo assimilável (P), não foram observadas diferenças entres os horizontes superficiais e subsuperficiais, com ampla variação dos valores entre 0 e 15 e, 0 e 11 mg kg1, respectivamente (Tabela 3 ). Diferentemente, os teores de Prem apresentam ampla variação e diferença estatística significativa entre as médias totais dos horizontes $\mathrm{A}$ e $\mathrm{B}+\mathrm{C}$, com maiores valores no horizonte $\mathrm{A}$, entre 13 e $31 \mathrm{mg} \mathrm{L}^{-1}$ (média 24) e entre 4 e $24 \mathrm{mg} \mathrm{L}^{-1}$ (média de 13) nos horizontes $\mathrm{B}+\mathrm{C}$ (Tabelas 1 e 2).

Em solos de diversas regiões do Brasil e formados sob diferentes materiais de origem, Valladares, Pereira e Anjos (2003) observaram teores de Prem entre 20,0 e $40,8 \mathrm{mg} \mathrm{L}^{-1}$ nos horizontes $\mathrm{A}$ e entre 7,8 e $35,7 \mathrm{mg} \mathrm{L}^{-1}$ nos horizontes $\mathrm{B}$, sendo que para ambos os horizontes, os menores teores foram observados no Latossolo Bruno formado a partir de rochas básicas e alcalinas e os maiores no Argissolo Amarelo originado de rochas sedimentares e sedimentos diversos. Em solos do estado de Minas Gerais, Souza et al. (2006) observaram teores entre 8,3 e $26,8 \mathrm{mg} \mathrm{L}^{-1}$ nos horizontes A, sendo os menores teores observados no Latossolo Vermelho e os maiores no Neossolo Quartzarênico. Eberhardt et al. (2008) quantificaram em Latossolos do cerrado nos horizontes A valores entre 5,2 e 40,8 $\mathrm{mg} \mathrm{L}^{-1}$. Em solos desenvolvidos sob rochas básicas-alcalinas e ultrabásicas no Alto Paranaíba, Rolim Neto et al. (2004) observaram ampla variação e teores entre 0 e $22 \mathrm{mg} \mathrm{L}^{-1}$ nos horizontes B de Latossolos e Cambissolos.

Para ambos os horizontes $\mathrm{A}$ e $\mathrm{B}+\mathrm{C}$, observase que os atributos relacionados à mineralogia se destacam como condicionantes da adsorção específica de fósforo, como observado pelos valores das correlações entre os teores de Prem e atributos químicos, físicos e mineralógicos analisados, sendo a magnitude de adsorção de fósforo entre horizontes superficiais e subsuperficiais observada pelas diferenças de correlações em cada horizonte. Neste sentido, destacam-se as correlações negativas do Prem nos horizontes A em ordem decrescente com $\mathrm{Fe}_{2} \mathrm{O}_{3}(\operatorname{raios} \mathrm{X})>\mathrm{Fed}>\mathrm{SE}>$ Feo $(-0,86 \mathrm{e}-0,71)$, enquanto, para os horizontes $\mathrm{B}+\mathrm{C}$ com $\mathrm{Fe}_{2} \mathrm{O}_{3}$ (ataque sulfúrico) $>\mathrm{Fe}_{2} \mathrm{O}_{3}($ raios $\mathrm{X})>$ argila $>\mathrm{Fed}>$ Feo $(-0,81$ e $-0,62)$ (Tabela 3$)$.

Tabela 3. Coeficiente de correlação simples entre Prem e atributos dos horizontes A ( $n=14)$ e $B+C(n=13)$.

\begin{tabular}{|c|c|c|c|c|c|}
\hline Atributos & A & $\mathrm{B}+\mathrm{C}$ & Atributos & $\mathbf{A}$ & $\mathrm{B}+\mathrm{C}$ \\
\hline pH (água) & $-0,32$ & $-0,58^{*}$ & $\mathrm{Al}_{2} \mathrm{O}_{3}{ }^{(2)}$ & $-0,27$ & $-0,54$ \\
\hline $\mathrm{Ca}^{2+}$ & $-0,54^{*}$ & $-0,57^{*}$ & $\mathrm{~F}_{2} \mathrm{O}_{3}^{(2)}$ & $-0,86^{*}$ & $-0,80 *$ \\
\hline $\mathrm{Mg}^{2+}$ & $-0,41$ & $-0,24$ & $\mathrm{Al}_{2} \mathrm{O}_{3}{ }^{(3)}$ & - & $-0,57^{*}$ \\
\hline $\mathrm{Al}^{3+}$ & 0,29 & $0,60 *$ & $\mathrm{~F}_{2} \mathrm{O}_{3}^{(3)}$ & - & $-0,81^{*}$ \\
\hline $\mathrm{CTC}^{(1)}$ & $-0,57^{*}$ & $-0,54$ & Fed $^{(4)}$ & $-0,84 *$ & $-0,63 *$ \\
\hline $\mathrm{COT}^{(1)}$ & $-0,25$ & 0,08 & $\mathrm{Feo}^{(4)}$ & $-0,71 *$ & $-0,62 *$ \\
\hline Argila & $-0,39$ & $-0,67 *$ & $\mathrm{SE}^{(1)}$ & $-0,75^{*}$ & $-0,59^{*}$ \\
\hline
\end{tabular}

${ }^{*}$ Indica probabilidade $\mathrm{p}<0,05$.

(1) CTC: capacidade de troca catiônica a pH 70; COT: carbono orgânico total; SE: superfície específica.

(2) Obtido pelo raio X por fluorescência;

(3) Obtido pelo ataque sulfúrico $(1: 1)$

(4) Fed: ferro extraído com ditionito-citrato-bicarbonato; Feo: ferro extraído com oxalato de amônio.

Fonte: Elaboração dos autores. 
As correlações negativas do Prem com pH (água), $\mathrm{Ca}^{2+}$ e CTC indicam que as reações que controlam a disponibilidade de fosfato estão relacionadas também a presença de cátions na solução do solo, principalmente em solos com altos teores de bases trocáveis, como o $\mathrm{Ca}^{2+}$ e $\mathrm{Mg}^{2+}$. Segundo Meurer, Rheinheimer e Bissani (2006), a presença dos cátions solúveis em altos teores potencializa a formação de fosfato insolúvel nos solos. Enquanto, a correlação positiva do Prem com $\mathrm{Al}^{3+}(0,60 *)$ nos horizontes $\mathrm{B}$ e $\mathrm{C}$ deve ser analisada como uma correlação espúria, estando a hipótese relacionada à superestimação dos teores de $\mathrm{Al}^{3+}$ nos horizontes Bi (T2P1) e Cg (T1P5 e T2P5) levando a inflexão positiva da linha de tendência. Nos horizontes pouco intemperizados (Bi), o cloreto de potássio utilizado como extrator provoca a dissolução das formas de alumínio de baixa cristalinidade e do alumínio interestratificado, que são formas não trocáveis (MARQUES et al., 2002; ABREU JUNIOR; MURAOKA; LAVORANTE, 2003). No horizontes $\mathrm{C}$ glei, os teores de $\mathrm{Al}_{2} \mathrm{O}_{3}$ e $\mathrm{Fe}_{2} \mathrm{O}_{3}$ (raios $\mathrm{X}$ e ataque sulfúrico) bastante inferiores aos demais horizontes Bw, Bt e Bi (Tabela 4), confere menor adsorção de fosfato.

Sob o aspecto das relações com atributos dos solos, Souza et al. (2006) observaram correlação negativa do Prem com os teores de $\mathrm{Al}_{2} \mathrm{O}_{3}$ e $\mathrm{Fe}_{2} \mathrm{O}_{3}$ (ataque sulfúrico), assim como, Eberhardt et al. (2008) correlações negativas do Prem com Feo e Alo $>$ Fed e Ald $>$ argila. Avaliando a relação entre a capacidade de adsorção de fósforo em diferentes solos, Valladares, Pereira e Anjos (2003) observaram correlações entre positivas nos horizontes A com $\mathrm{SE}>$ argila $>\mathrm{Al}_{2} \mathrm{O}_{3}$ (ataque sulfúrico) $=$ Fed e nos horizontes $\mathrm{B}$ com $\mathrm{Al}_{2} \mathrm{O}_{3}$ (ataque sulfúrico) $>\mathrm{SE}>$ argila $>$ Fed $>\mathrm{Fe}_{2} \mathrm{O}_{3}$ (ataque sulfúrico), assim como, Gonçalves et al. (2011) verificou correlação na seguinte sequência $\mathrm{Feo}>\mathrm{Fed}>$ argila.
Para horizontes B, Rolim Neto et al. (2004) observaram correlação negativa entre a capacidade de adsorção e Fed, enquanto, Vilar et al. (2010) verificaram correlação positiva com Fed. Em estudo detalhado com as frações de fósforo nos solos, Almeida, Torrent e Barrón (2003) observaram em Latossolos desenvolvidos de basalto nos estados de Santa Catarina e Rio Grande do Sul menores teores de fósforo na superfície dos óxidos de maior cristalinidade, identificados pelos maiores teores de Fed.

Diferenças também foram observadas quando feita a análise individual de cada topossequência, sendo os maiores teores de Prem verificados no horizonte A (Tabela 2). Os maiores teores de Prem nestes horizontes são atribuídos aos menores teores de argila, $\mathrm{Al}_{2} \mathrm{O}_{3}$ e $\mathrm{Fe}_{2} \mathrm{O}_{3}$ (raios X), Fed e SE (Tabela 5), uma vez que os atributos relacionados com o Prem são os mesmos para os horizontes A e B+C, como observados pelas correlações negativas na Tabela 3.

Entre as topossequências, a T2 apresentou os maiores teores Prem no horizonte A, enquanto nos horizontes $\mathrm{B}+\mathrm{C}$ houve diferença entre $\mathrm{T} 2$ e $\mathrm{T} 3$ e intermediário para $\mathrm{T} 1$, com ampla variação dos teores de Prem nas T1 e T2 (Tabela 2). Verificase que, entre os atributos relacionados à adsorção de fósforo, a T2 apresentou nos horizontes $\mathrm{A}$ os menores teores de $\mathrm{Fe}_{2} \mathrm{O}_{3}$ (raios $\mathrm{X}$ e ataque sulfúrico), Fed, Feo e $\mathrm{SE}$ e, nos horizontes $\mathrm{B}+\mathrm{C}$ os menores teores de $\mathrm{Fe}_{2} \mathrm{O}_{3}$ (raios $\mathrm{X}$ e ataque sulfúrico) e $\mathrm{SE}$ (Tabela 5). Estes atributos estão correlacionados negativamente com os teores de Prem (Tabela 3). Na T2 ainda, destaca-se os baixos teores de argila nos horizontes $\mathrm{A}$, associados à formação do horizonte B textural nos Argissolos e do baixo grau de desenvolvimento dos Cambissolos (Tabela 4). 


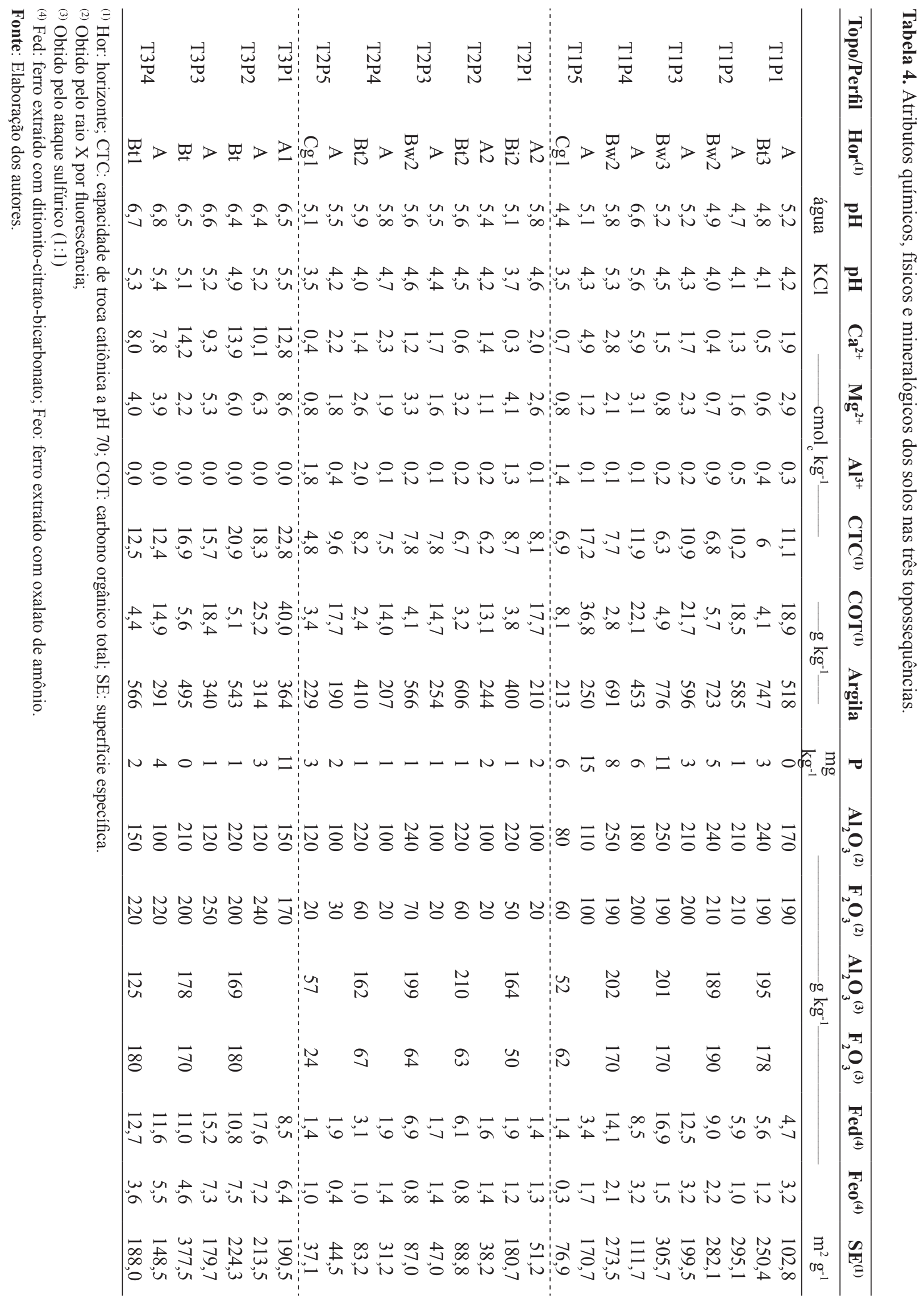




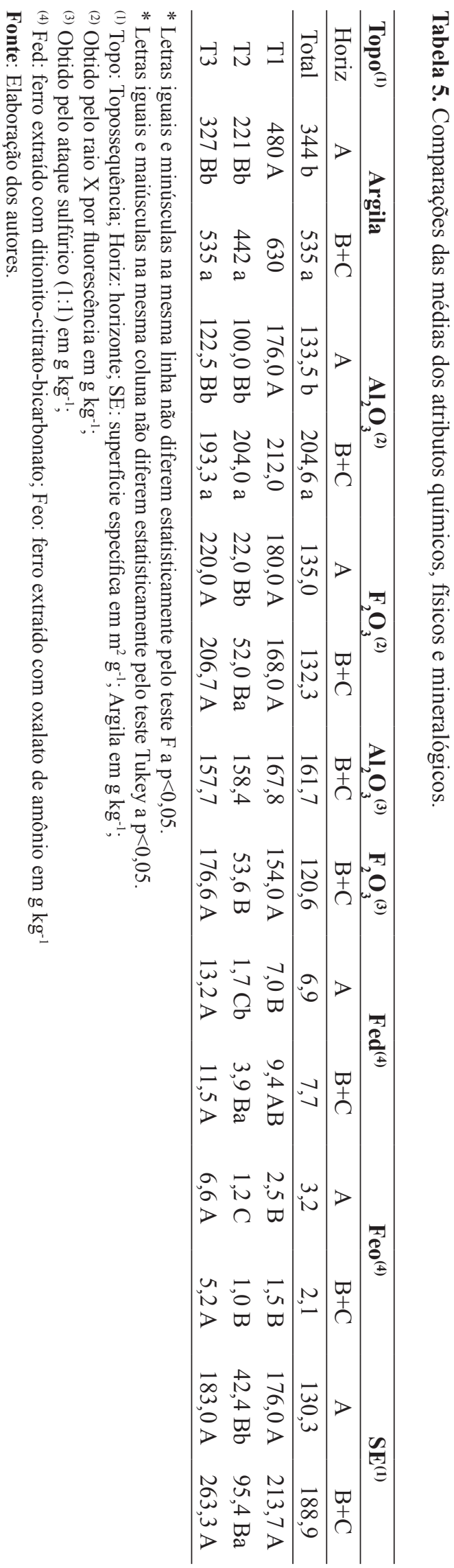

Associado ainda à adsorção de fósforo nos solos observa-se na composição mineralógica da fração argila dos horizontes $\mathrm{A}$ e $\mathrm{B}+\mathrm{C}$ o predomínio de minerais $1: 1$ (caulinita) e óxido de ferro (goethita) na $\mathrm{T} 1$ e $\mathrm{T} 2$, tendo a $\mathrm{T} 2$ presença de minerais primários (mica), enquanto, minerais 1:1 (caulinita) e 2:1 (illita-montmorilonita) na T3. Já, na fração óxidos, os horizontes $\mathrm{B}+\mathrm{C}$ apresentam o predomínio de goethita, seguida de hematita, além de outros minerais na T3 (Tabela 6). Assim, as diferenças nos teores de $\mathrm{Fe}_{2} \mathrm{O}_{3}$, Fed, Feo e SE destacados nas Tabelas 4 e 5, são o reflexo da maior ou menor participação do óxido de ferro goethita, principalmente.

Segundo Bahia Filho et al. (1983) a goethita é considerada a principal responsável pelo fenômeno de adsorção específica de fósforo nos solos do Brasil Central. Outros trabalhos também tem apresentado maior capacidade de adsorção de fósforo em solos com maior teor de goethita quando comparada a hematita (BIGHAM et al., 1978; JONES, 1981; KARIM; ADAMS, 1984; FONTES; WEED, 1996). Eberhardt et al. (2008) observaram em Latossolos do cerrado correlações negativas e significativas entre a gibbsita, goethita e hematita com Prem $(-0,64,-0,64$ e $-0,43$, respectivamente), enquanto a caulinita não exerceu influência sobre o Prem $(-0,17)$. Segundo Fontes e Weed (1996) em Latossolos brasileiros a gibbsita, goethita e hematita, explicam mais de 95 $\%$ da variação na adsorção máxima de fosfato.

Em Latossolos do Estado de São Paulo, Alves e Lavorenti (2004), observaram maiores valores de correlação negativa da goethita em relação à hematita com os teores de Prem, estando essa maior adsorção relacionada às suas propriedades intrínsecas, tais como o menor grau de cristalinidade, maior substituição do Al, maior superfície específica e maior quantidade de faces expostas para adsorção (ALVES; LAVORENTI, 2004). Essa maior capacidade de adsorção dos solos goethíticos foi também constatada por Curi e Franzmeier (1984), sendo creditada à facilidade de acesso do fosfato aos grupos OH- de superfície (FROSSARD et al., 1994), 
uma vez observada maior área superficial específica (TORRENT; SCHWERTMANN, BARRÓN, 1994; ROLIM NETO et al., 2004).

Entretanto Torrent, Schwertmann e Barrón (1992; 1994), encontraram valores médios de adsorção de fósforo para goethitas similares aos de hematitas, mas a variabilidade entre as amostras de hematita foi muito maior. Tais variações podem ser resultantes de diferenças nas características estruturais, relacionadas com as dimensões dos cristais, faces do cristal e presença de domínios na estrutura interna dos mesmos, que podem favorecer maior ou menor adsorção (TORRENT, 1997).

Tabela 6. Composição mineralógica da fração argila e óxidos.

\begin{tabular}{ccc}
\hline Topossequência & Argila & Óxidos \\
\hline & $\mathrm{A}, \mathrm{B}+\mathrm{C}$ & $\mathrm{B}+\mathrm{C}$ \\
\hline $\mathrm{T} 1$ & caulinita $>>$ goethita & goethita $>$ hematita $>$ ilmenita \\
$\mathrm{T} 2$ & caulinita $>>$ mica $>$ goethita & goethita $>$ hematita $>$ caulinita $>$ maghemita/magnetita \\
T3 & caulinita $>>$ illita-montmorilonita & goethita $>$ hematita $>$ ilmenita $>$ ferrihidrita/maghemita \\
\hline
\end{tabular}

Fonte: Elaboração dos autores.

Outras variações podem estar relacionadas com a presença de outros minerais da fração óxidos, como a presença de maghemita, magnetita e illmenita, as quais também contribuem para maior adsorção de fósforo (SOUZA; LOBATO, 2004). A presença de maghemita tem se mostrado comum em vários solos, principalmente naqueles derivados de rochas vulcânicas básicas (COSTA et al., 1999).

Pelos dados da distribuição dos atributos na análise multivariada de componentes principais, observou-se que os teores de Prem e os atributos que representam a mineralogia dos solos estão dispostos de forma antagônica, evidenciando a capacidade dos óxidos de ferro e alumínio na adsorção específica de fósforo, tanto nos horizontes A como $\mathrm{B}+\mathrm{C}$ (Figura 1ab). Padrão semelhante da distribuição dos atributos foi observado por Eberhardt et al. (2008) em horizontes A de Latossolos do cerrado, antagônica com a mineralogia, e correlação negativa com atributos $\mathrm{pH}$ (água), $\mathrm{Ca}^{2+}, \mathrm{Mg}^{2+}$ e $\mathrm{SB}$ e perpendicular ao $\mathrm{Al}^{3+}$.

Pela análise de agrupamentos hierárquicos, observa-se pelo dendograma nos horizontes A três grupos em relação à similaridade (Figura 2a), destacando os agrupamentos dos perfis de acordo com os materiais de origem: grupo I, perfis sob basalto; grupo II, perfis sob muscovita-biotitagnaisse e grupo III, perfis sob gabro e o perfil T1P5 (basalto). A presença do perfil T1P5 no grupo III, indica que a localização na baixada com recebimento de sedimentos o aproxima dos perfis sob gabro, como observado pelos teores de $\mathrm{Ca}^{2+}$, argila, $\mathrm{Al}_{2} \mathrm{O}_{3}$ (raios X) e CTC.

No dendograma dos horizontes $\mathrm{B}+\mathrm{C}$ (Figura 2b), destacam-se quatro grupos em relação à similaridade, destacando: grupo I, perfis sob basalto; grupo II, perfis sob muscovita-biotita-gnaisse; grupo III, perfis sob gabro e grupo IV, representado pelos perfis T1P5 e T2P5, com subsuperficiais C glei, estando localizados na parte baixa da paisagem e com padrões diferenciados dos demais, com baixos teores de $\mathrm{Al}_{2} \mathrm{O}_{3}$ e $\mathrm{Fe}_{2} \mathrm{O}_{3}$ (raios X), menores superfície específica, teores de Fed, Feo e argila, e maiores teores de $\mathrm{Al}^{3+}$ e Prem. 
Figura 1. Dispersão dos atributos pela análise de componentes principais dos horizontes $A$ (a) e $B+C$ (b).
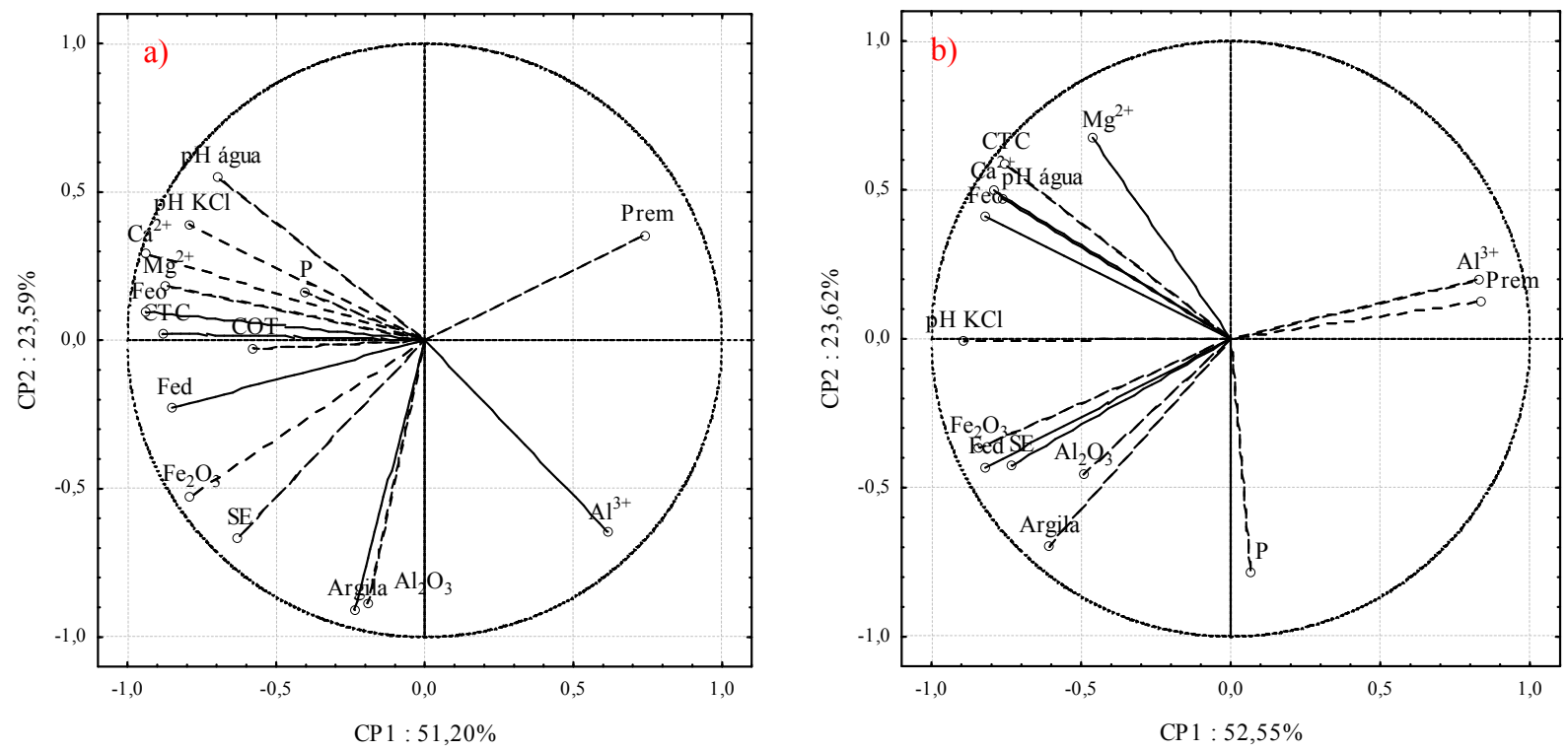

Fonte: Elaboração dos autores.

Figura 2. Agrupamento das topossequências e perfis para os horizontes A (a) e B+C (b).
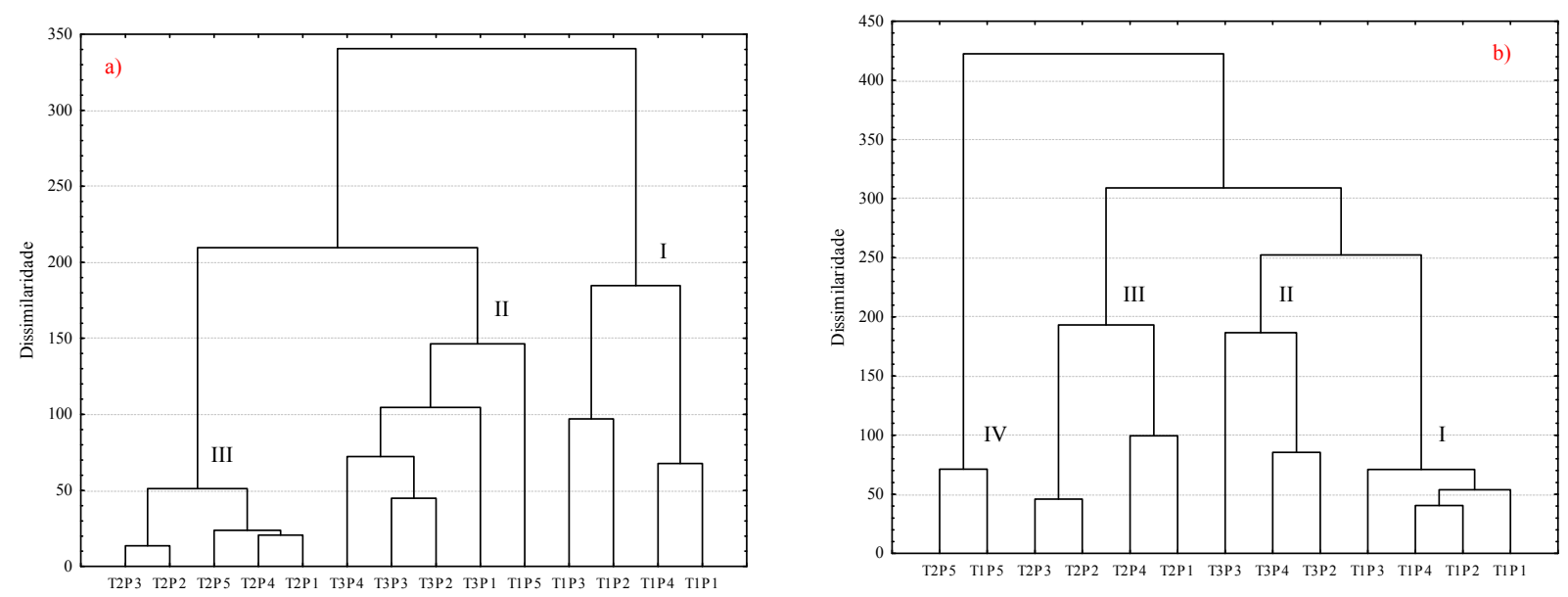

Fonte: Elaboração dos autores.

\section{Conclusões}

Os teores de Prem apresentaram em ordem decrescente da topossequência sob muscovitabiotita-gnaisse, basalto, gabro no horizonte A e da topossequência sob basalto, muscovita-biotitagnaisse, gabro nos horizontes $\mathrm{B}+\mathrm{C}$.
No horizonte A foi observado correlações negativas e significativas do Prem em ordem decrescente com $\mathrm{Fe}_{2} \mathrm{O}_{3}$ (raios X), Fed, SE e Feo e nos horizontes $\mathrm{B}+\mathrm{C}$ com $\mathrm{Fe}_{2} \mathrm{O}_{3}$ (ataque sulfúrico), $\mathrm{Fe}_{2} \mathrm{O}_{3}$ (raios X), argila, Fed e Feo. 
Pela análise multivariada de componentes principais observou-se que os teores de Prem e os atributos que representam a mineralogia dos solos dispostos de forma antagônica, evidenciando a capacidade dos óxidos de fero e alumínio na adsorção específica de fósforo.

Pela análise de agrupamentos hierárquicos observou-se nos horizontes A três grupos, destacando os agrupamentos dos perfis de acordo com os materiais de origem, sendo exceção o T1P5 com os perfis sob gabro e nos horizontes $\mathrm{B}+\mathrm{C}$ quatro grupos, com separação dos perfis T1P5 e T2P5 que apresentam horizontes $\mathrm{C}$ glei.

\section{Agradecimentos}

Ao Curso de Pós-Graduação em Agronomia - Ciência do Solo da Universidade Federal Rural do Rio de Janeiro, a Facultad de Ciencias da Universidad de Granada.

\section{Referências}

ABREU JUNIOR, C. H.; MURAOKA, T.; LAVORANTE, A. F. Exchangeable aluminum evaluation in acid soils. Scientia Agrícola, Piracicaba, v. 60, n. 3, p. 543-548, 2003.

ALCARDE, J. C.; GUIDOLIN, J. A.; LOPES, A. S. Os adubos e a eficiência das adubações. 2. ed. São Paulo: ANDA, 1991. p. 11-18. (Boletim técnico, 3).

ALMEIDA, J. A.; TORRENT, J.; BARRÓN, V. Cor do solo, formas do fósforo e adsorção de fosfatos em Latossolos desenvolvidos de basalto do extremo sul do Brasil. Revista Brasileira de Ciência do Solo, Viçosa, v. 27, n. 3, p. 985-1002, 2003.

ALVAREZ V., V. H.; FONSECA, D. M. Definição de doses de fósforo para determinação da capacidade máxima de adsorção de fosfatos e para ensaios em casa de vegetação. Revista Brasileira de Ciência do Solo, Campinas, v. 14, n. 1, p. 49-55, 1990.

ALVAREZ V., V. H.; NOVAIS, R. F.; DIAS, L. E.; OLIVEIRA, J. A. Determinação e uso do fósforo remanescente. Sociedade Brasileira de Ciência do Solo. Boletim Informativo, Viçosa, v. 25, n. 1, p. 27-33, 2000.
ALVES, M. E.; LAVORENTI, A. Remaining phosphorus and sodium fluoride $\mathrm{pH}$ in soils with different clay contents and clay mineralogies. Pesquisa Agropecuária Brasileira, Brasília, v. 39, n. 3, p. 241-246, 2004.

ANDRADE, F. V.; MENDONÇA E. S.; ALVAREZ V. H.; NOVAIS, R. F. Adição de ácidos orgânicos e húmicos em Latossolos e adsorção de fosfato. Revista Brasileira de Ciência do Solo, Viçosa, v. 27, n. 6, p. 1003-1011, 2003.

ANTELO, J.; ARCE, F.; AVENA, M.; FIOL, S.; LÓPEZ, R.; MACÍAS, F. Adsorption of soil humic acid at the surface of goethite and its competitive interaction with phosphate. Geoderma, Amsterdam, v. 138, n. 1, p. 12-19, 2007.

BACHE, B. W.; WILLIAMS, E. G. A phosphate sorption index for soils. European Journal of Soil Science, Oxford, v. 22, n. 3, p. 289-301, 1971.

BAHIA FILHO, A. F. C.; BRAGA, J. M.; RESENDE, M.; RIBEIRO, A. C. Relação entre adsorção de fósforo e componentes mineralógicos da fração argila de Latossolos do Planalto Central. Revista Brasileira de Ciência do Solo, Campinas, v. 7, n. 1, p. 221-226, 1983.

BIGHAM, J. M.; GOLDEN, D. C.; BUOL, S. W.; WEED, S. B.; BOWEN, L. H. Iron oxide mineralogy of well-drained Ultisols and Oxisols: II. Influence on color, surface area, and phosphate retention. Soil Science Society of American Journal, Madison, v. 42, n. 5, p. 825 830, 1978.

BRAGA, J. M.; DEFELIPO, B. V. Relações entre formas de fósforo inorgânico, fósforo disponível e material vegetal em solos sob vegetação de cerrado: I - trabalhos de laboratório. Ceres, São Paulo, v. 19, n. 1, p. 124-136, 1972.

COSTA, A. C. S.; BIGHAM, J. M.; RHOTON, F. E.; TRAINA, S. J. Quantification and characterization of maghemite in soils derived from volcanic rocks in southern Brazil. Clays Clay Minerals, Urbana, v. 47, n. 4, p. 466-473, 1999.

CURI, N.; FRANZMEIER, D. P. Toposequence of oxisols from the central plateau of Brazil. Soil Science Society of American Journal, Madison, v. 48, n. 4, p. 341346, 1984.

DYAL, R. S.; HENDRICKS, S. B. Total surface of clays in polar liquids as a characteristic index. Soil Science, Philadelphia, v. 69, n. 6, p. 503-509, 1950.

EBERHARDT, D. N.;VENDRAME,P.R. S.;BECQUER, T.; GUIMARÃES, M. F. Influência da granulometria e da mineralogia sobre a retenção do fósforo em Latossolos sob pastagens no cerrado. Revista Brasileira de Ciência do Solo, Viçosa, v. 32, n. 3, p. 1009-1016, 2008. 
EMPRESA BRASILEIRA DE PESQUISA AGROPECUÁRIA - EMBRAPA. Manual de métodos de análise de solos. Rio de Janeiro: Embrapa, 1997. 212 p.

FONTANA, A.; PEREIRA, M. G.; SALTON, J. C.; LOSS, A.; CUNHA, T. J. F. Fósforo remanescente e correlação com as substâncias húmicas em um Latossolo Vermelho sob diferentes sucessões de cultura em plantio direto. Revista Brasileira de Agrociência, Pelotas, v. 14, n. 1, p. 161-166, 2008.

FONTES, M. P. F.; WEED, S. B. Phosphate adsorption by clays from Brazilian oxisols: relationships with specific surface area and mineralogy. Geoderma, Amsterdam, v. 72, n. 1, p. 37-51, 1996.

FROSSARD, E.; BROSSARD, M.; HEDLEY, M. J.; METHERELL, A. Reactions controlling the cycling of $P$ in soils. In: TIESSEN, H. (Ed.). P cycling in terrestrial and aquatic ecosystem; a global perspective. New York: John Wiley, 1994. p. 1-65.

GONÇALVES, G. K.; MEURER, E. J.; BORTOLON, L.; GONÇALVES, D. R. N. Relação entre óxidos de ferro e de manganês e a sorção de fósforo em solos no Rio Grande do Sul. Revista Brasileira de Ciência do Solo, Viçosa, v. 35, n. 6, p. 1633-1639, 2011.

HALLETT, R. B.; KYLE, P. R. XRF and INAA determinations of major and trace elements in geological survey of Japan igneous and sedimentary rock standards. Geostandards Newsletter, Malden, v. 17, n. 1, p. 127133, 1993.

HEILMAN, M. D.; CARTER, D. L.; GONZALEZ, C. L. The ethylene glycol monoethyl ether technique for determining soil surface area. Soil Science, Philadelphia, v. 100, n. 6, p. 409-413, 1965.

JONES, R. C. X-ray diffraction line profile analysis vs. phosphorus sorption by 11 Puerto Rican soils. Soil Science Society of American Journal, Madison, v. 45, n. 4, p. 818-825, 1981.

KÄMPF, N.; SCHWERTMANN, U. The $5 \mathrm{M} \mathrm{NaOH}$ concentration treatment for iron oxides in soils. Clays Clay Minerals, Urbana, v. 30, n. 6, p. 400-408, 1982.

KARIM, M. I.; ADAMS, W. A. Relationships between sesquioxides, kaolinite, and phosphate sorption in a catena of Oxisols in Malawi. Soil Science Society of American Journal, Madison, v. 48, n. 2, p. 406-409, 1984.

MARQUES, J. J.; TEIXEIRA, W. G.; SCHULZE, D. G.; CURI, N. Mineralogy of soils with unusually high exchangeable $\mathrm{Al}$ from the western Amazon Region. Clay Minerals, v. 37, n. 4, p. 651-661. 2002.
MEHRA, O. P.; JACKSON, M. L. Iron oxides removal from soils and clays by a dithionite-citrate-bicarbonate system buffered with sodium bicarbonate. Clays Clay Minerals, Urbana, v. 7, n. 1, p. 317-327, 1960.

MEURER, E. J.; RHEINHEIMER, D.; BISSANI, C. A. Fenômenos de sorção em solos. In: MEURER, E. J. (Ed.). Fundamentos de química do solo. 3. ed. Porto Alegre: Evangraf, 2006. 285 p.

NORRISH, K.; TAYLOR, R. M. The isomorphous replacement of iron by aluminum in soil goethites. European Journal of Soil Science, Oxford, v. 12, n. 2, p. 294-306, 1961.

NOVAIS, R. F.; SMYTH, T. J. Fósforo em solo e planta em condições tropicais. Viçosa: Universidade Federal de Viçosa, 1999. 399 p.

PARFITT, R. L. Anion adsorption by soil and soil materials. Advances in Agronomy, v. 30, p. 1-50, 1978.

ROLIM NETO, F. C.; SCHAEFER, C. E. G. R.; COSTA, L. M.; CORRÊA, M. M.; FERNANDES FILHO, E. I.; IBRAIMO, M. M. Adsorção de fósforo, superfície específica e atributos mineralógicos em solos desenvolvidos de rochas vulcânicas do alto Paranaíba (MG). Revista Brasileira de Ciência do Solo, Viçosa, v. 28, n. 6, p. 953-964, 2004.

SANTOS, R. D.; LEMOS, R. C.; SANTOS, H. G.; KER, J. C. ANJOS, L. H. C. Manual de descrição e coleta de solo no campo. 5. ed. Viçosa, MG: SBCS/EMBRAPACNPS, 2005. $100 \mathrm{p}$.

SCHULZE, D. G. The influence of aluminum on iron oxides. VII. Unit-cell dimensions of al-substituted goethites and estimation of $\mathrm{Al}$ from them. Clays Clay Minerals, Urbana, v. 32, n. 1, p. 27-39, 1984.

SCHWERTMANN, U. Differenzierung der eisenoxide des bodens durch photochemische extraktion mit saurer ammoniumoxalate-Lösung. Zeitschrift für Pflanzenernährung, Düngung, Bodenkunde, v. 105, n. 1, p. 194-202, 1964.

SOUZA, D. M. G.; LOBATO, E. Cerrado: correção do solo e adubação. 2. ed. Brasília: Embrapa Informação Tecnológica, 2004. 416 p.

SOUZA, R. F.; FAQUIN, V.; TORRES, P. R. F.; BALIZA, D. P. Calagem e adubação orgânica: influência na adsorção de fósforo em solos. Revista Brasileira de Ciência do Solo, Viçosa, v. 30, n. 6, p. 975-983, 2006.

SPOSITO, G. The chemistry of soils. New York: Oxford University Press, 1989. 277 p.

TORRENT, J. Interactions between phosphate and iron oxide. Advanced Geoecology, v. 30, p. 321-344, 1997. 
TORRENT, J.; SCHWERTMANN, U.; BARRÓN, V. Fast and slow phosphate sorption by goethite-rich natural materials. Clays Clay Minerals, Urbana, v. 40, n. 1, p. 14-21, 1992.

Phosphate sorption by natural hematites. European Journal of Soil Science, Oxford, v. 45, n. 1, p. 45-51, 1994.
VALLADARES, G. S.; PEREIRA, M. G.; ANJOS, L. H. C. Adsorção de fósforo em solos de argila de atividade baixa. Bragantia, Campinas, v. 62, n. 1, p. 111-118, 2003.

VILAR, C. C.; COSTA, A. C. S.; HOEPERS, A.; SOUZA JUNIOR, I. G. Capacidade máxima de adsorção de fósforo relacionada a formas de ferro e alumínio em solos subtropicais. Revista Brasileira de Ciência do Solo, Viçosa, v. 34, n. 4, p. 1059-1068, 2010. 